\title{
Coordination Chemistry of Perhalogenated Cyclopentadienes and Alkynes. 8. ${ }^{1}$ Pentakls(dlmethylsilyl)cymantrene ${ }^{\dagger}$
}

\author{
Karlheinz Sünkel" and Jullan Hofmann \\ Insthut fur Anorganische Chemie der Universftät, Molserstrasse 1, D-8000 Munich 2, Germany \\ Received June 8, 1992
}

\begin{abstract}
Summary: Starting from $\left[\eta^{5}-\mathrm{C}_{5} \mathrm{Br}_{5}\right] \mathrm{Mn}(\mathrm{CO})_{3}$, the first $\pi$-complex of a cyclopentadienyl ligand with five slyl substituents, $\left[\eta^{5}-\mathrm{C}_{5}\left(\mathrm{SiM}_{2} \mathrm{H}\right)_{5}\right] \mathrm{Mn}(\mathrm{CO})_{3}$, was prepared by a series of halogen-lithium exchange reactions, followed by silylation with $\mathrm{SiMe}_{2} \mathrm{HCl}$. The crystal structure determination of this compound shows a highly symmetrical "paddle wheel" orientation of the flve silyl groups around the cyclopentadlenyl ring.
\end{abstract}

\section{Introduction}

Polysilylated cyclopentadienyl ligands have been of interest over the past decade. Increasing the number of silyl substituents is believed to enhance the solubility in nonpolar solvents and the volatility of the compounds and to increase their kinetic stability. Thus, a series of stable $\pi$-cyclopentadienyl complexes of main-group elements and lanthanides or actinides with the $\left[\mathrm{C}_{5}\left(\mathrm{SiMe}_{3}\right)_{3} \mathrm{H}_{2}\right]$ ligand could be obtained. ${ }^{2}$ However, attempts to introduce more than three $\mathrm{SiMe}_{3}$ groups into the cyclopentadienyl system thus far have been unsuccessful. ${ }^{3}$ We report here the first $\pi$-complex containing a 5-fold silylated cyclopentadienyl ligand.

\section{Results and Discussion}

We showed recently that starting from $\left[\left(\mathrm{C}_{5} \mathrm{Cl}_{4} \mathrm{Br}\right) \mathrm{Mn}\right.$ $(\mathrm{CO})_{3}$ ] (1), the consecutive introduction of five SMe groups into the cyclopentadienyl ligand can be achieved by repeated alternate reaction with $n$-butyllithium and dimethyl disulfide. ${ }^{4}$ When other electrophiles are used, multiple substitution in 1 works only when electrophiles containing donor atoms such as $\mathrm{S}, \mathrm{Se}$, or $\mathrm{P}$ are used. However, in the case of the related $\left[\left(\mathrm{C}_{5} \mathrm{Br}_{5}\right) \mathrm{Mn}(\mathrm{CO})_{3}\right](2)^{5}$ all the bromine atoms can be substituted stepwise by the $\mathrm{SiMe}_{2} \mathrm{H}$ group (Scheme I).

Double lithiations can be performed for each of the compounds 2-5. Thus, 7 can be prepared in three steps from 2 in an overall yield of about $60 \%$. All derivatives $\left[\mathrm{C}_{5} \mathrm{Br}_{5-n}\left(\mathrm{SiMe}_{2} \mathrm{H}\right)_{n}\right] \mathrm{Mn}(\mathrm{CO})_{3}$ with $n=1-5$ are air-stable, crystalline powders, that are very soluble in hexane and can be obtained in good to excellent yields. Their ${ }^{1} \mathrm{H}$ NMR spectra show the minimum number of signals that can be expected from symmetry considerations. This means obviously that in all compounds, even in the sterically crowded 6 and 7 , a rapid rotation of the silyl group around the ring-silicon bond must occur.

Comparable sterically congested systems such as $\mathrm{Cr}$ (CO) ${ }_{3}\left[\mathrm{C}_{6}\left(\mathrm{SiMe}_{2} \mathrm{H}\right)_{6}\right]{ }^{6}\left[\mathrm{C}_{5}\left({ }^{i} \mathrm{Pr}\right)_{5}\right] \mathrm{CoCp}^{+}$, and $\left[\mathrm{C}_{5}\left({ }^{(} \mathrm{Pr}\right)_{5}\right]$ -

'Dedicated to Prof. Wolfgang Beck on the orcasion of his 60th birthday.

(1) Part 7: Sünkel, K. Chem. Ber. 1991, 124, 2449.

(2) For a review, see: Jutzi, P. J. Organomet. Chem. 1990, 400, 1, and literature cited therein.

(3) The synthesis of $\mathrm{C}_{8}\left(\mathrm{SiMe}_{3}\right)_{6}$ was reported in a short communication: Miftalkhof, M. S.; Tolstikov, G. A.; Lomailina, S. I. Zh. Obshch. Khim. 1976, 46, 2754; Chem. Abstr. 1977, 86, 1214302.

(4) Sünkel, K.; Motz, D. Angew. Chem., Int. Ed. Engl. 1988, $27,934$.

(5) Nefedov, V. A. Zh. Org. Khim. 1973, 9, 719. Herrmann, W. A., Huber, M. J. Organomet. Chem. 1977, 140, 55.

(6) Schuster, I. I.; Weissensteiner, W.; Mislow, K. J. Am. Chem. Soc. 1986, 108, 6661.

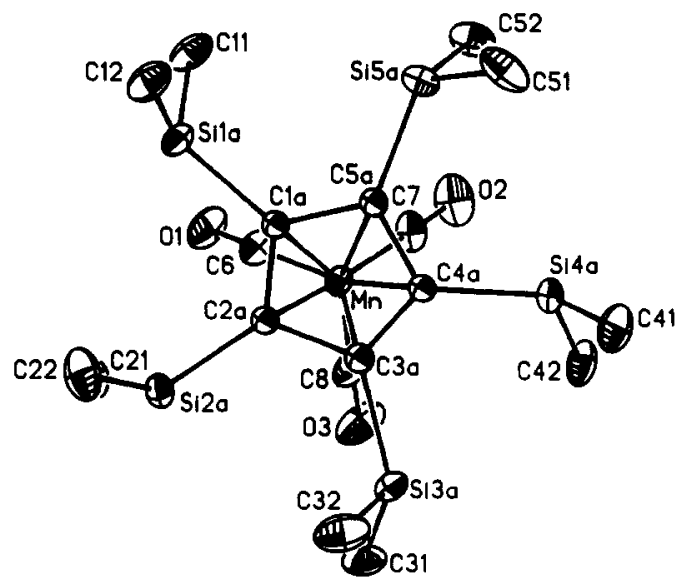

Figure 1. Molecular structure of 7 (thermal ellipsoids on the $20 \%$ probability level; the picture shows the higher populated A form). Averaged bond lengths $(\AA): \mathrm{Mn}-(\mathrm{C} 1 \mathrm{~A}-\mathrm{C} 5 \mathrm{~A}) 2.17$ (1); C-C 1.46 (1); $\mathrm{C}_{\text {ring }}-\mathrm{Si} 1.87$ (1); $\mathrm{Si}-\mathrm{C}_{\mathrm{Me}} 1.88$ (1).

$\mathrm{Mo}(\mathrm{CO})_{3} \mathrm{Me}^{8}$ show strongly temperature-dependent $\mathrm{NMR}$ spectra with internal rotation barriers ranging from 56 to $72 \mathrm{~kJ} / \mathrm{mol}$. Cooling a toluene solution of 7 results in broadening of the ${ }^{1} \mathrm{H}$ NMR doublet for the methyl groups until one broad resonance is observed at $-80^{\circ} \mathrm{C}$. Going down to $-90^{\circ} \mathrm{C}$, which is the lowest reasonable temperature achievable in this solvent, results in two broad signals without any fine structure. This leads to an approximate $\Delta G^{*}$ value of $40 \mathrm{~kJ} / \mathrm{mol}$. In comparison to the abovementioned compounds, this is a much lower value which means the steric hindrance is here not as great as might have been expected.

Figure 1 shows the solid state structure of $7 .^{9}$ All $\mathrm{SiMe}_{2} \mathrm{H}$ groups show the same "paddle wheel" orientation with the $\mathrm{Si}-\mathrm{H}$ bond lying in the plane of the cyclopentadienyl ring. Compound $\mathbf{7}$ is therefore chiral. Interestingly, there is an obvious disorder in the silicon positions, which also can be resolved for the ring carbon atoms but not for the methyl groups. The site occupancy factors can be refined to values of $0.74: 0.26$, which means that both enantiomers are distributed statistically over the four symmetry-equivalent positions of the unit cell and not in special pairs, as would be demanded by crystallographic symmetry. Similar observations have been reported for

(7) Gloaguen, B.; Astruc, D. J. Am. Chem. Soc. 1990, 112, 4607.

(8) Sitzmann, H. Chem. Ber. 1990, 123, 2311.

(9) In the first difference Fourier synthesis after the whole molecule had been identified, five maxima of electron density were found in a distance of about $1 \mathrm{~A}$ from each of the Si atoms in the plane of the cyclopentadienyl ring. Refinement of these positions as site $B$ for the silicon atoms lead to relative site occupancy factors of $3: 1$ for $A: B$, but no new positions for the corresponding five ring $C$ atoms could be located. However, the appearance of the corresponding thermal ellipsoids indicated that there were two carbon positions as well. By firing the sof of the found carbon sites to 0.5 and the isotropic thermal parameters to $0.02 \mathrm{~A}^{2}$, a new difference Fourier synthesis could localize the $\mathrm{B}$ site for the ring carbons as well. Isotropic refinement of the $\mathrm{C}$ and $\mathrm{Si}$ positions with the restriction of common thermal parameters for each A-B pair yielded a final sof of 0.74 for site $A$. No B sites for the methyl carbons could be localized; nor could any hydrogen positions. Thus, only one methyl carbon site was used in refinement and hydrogens were omitted at all. 
Scheme I ${ }^{a}$

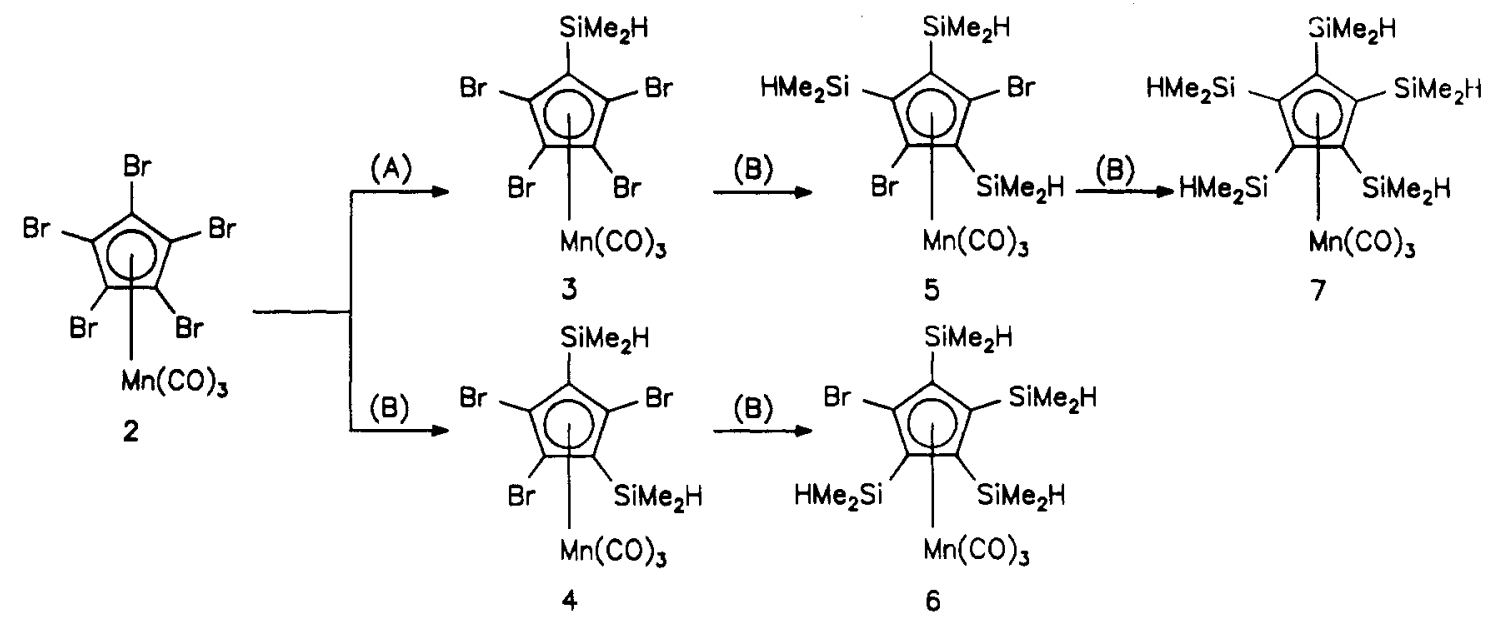

a (A) (1) 1.1 equiv of $n$-BuLi; (2) 1 equiv of $\mathrm{SiMe}_{2} \mathrm{HCl}$. (B) (1) 2 equiv of $n$-BuLi; (2) 2 equiv of $\mathrm{SiMe}_{2} \mathrm{HCl}$.

Table I. Crystallographic Data for 7

\begin{tabular}{|c|c|c|c|}
\hline & $\overline{\text { Cry }}$ & ters & \\
\hline $\begin{array}{l}\text { formula } \\
\text { space group } \\
\text { cryst syst } \\
a, \AA \\
b, \AA \\
c, \AA \\
V, \AA^{3}\end{array}$ & $\begin{array}{l}\mathrm{C}_{18} \mathrm{H}_{35} \mathrm{MnO}_{3} \mathrm{Si}_{5} \\
\text { Pna2 }_{1} \\
\\
\text { orthorhombic } \\
19.329(13) \\
10.693(6) \\
13.200(3) \\
2728(3)\end{array}$ & $\begin{array}{l}Z \\
\text { cryst dimens, } \\
\quad \mathrm{mm} \\
\text { cryst color } \\
D(\text { calc }), \mathrm{g} / \mathrm{cm}^{3} \\
\mu(\mathrm{Mo} \mathrm{K} \alpha), \mathrm{cm}^{-1} \\
\text { temp, }{ }^{\circ} \mathrm{C}\end{array}$ & $\begin{array}{l}4 \\
0.50 \times 0.50 \\
\quad \times 0.15 \\
\text { yellow } \\
1.119 \\
6.91 \\
18\end{array}$ \\
\hline $\begin{array}{l}\text { diffractometer } \\
\text { mono- } \\
\quad \text { chromator } \\
\text { scan technique } \\
\text { radiation } \\
\lambda, \AA \\
2 \theta \text { range, deg } \\
\text { std rflns }\end{array}$ & $\begin{array}{l}\quad \text { Data Col } \\
\text { Nicolet R3 } \\
\text { graphite } \\
\\
\omega-2 \theta \\
\text { Mo K } \alpha \\
0.71073 \\
2-20 \\
20-50 \\
2 \text { std } / 98 \text { rflns }\end{array}$ & $\begin{array}{l}\text { ction } \\
\text { rflns colled } \\
\text { indpt rflns } \\
R(\text { merg), \% } \\
\text { indpt rflns obsd } \\
F_{\text {o }} \geq 4 \sigma\left(F_{0}\right) \\
\text { data collcd }\end{array}$ & $\begin{array}{l}3955 \\
2670 \\
3.98 \\
2308 \\
\\
\pm h, \pm k, \pm l \\
+h,+k,+l\end{array}$ \\
\hline $\begin{array}{l}\text { program } \\
\text { abs corr } \\
\text { min/max } \\
\text { transm }\end{array}$ & $\begin{array}{l}\text { Refinem } \\
\text { SHELXTL PLUS } \\
4.11 / V \\
\text { empirical ( } \psi \text { scan) } \\
0.388 / 0.502\end{array}$ & & \\
\hline $\begin{array}{l}R, \% \\
\Delta_{\max }(\rho), \text { e } \AA^{-3}\end{array}$ & $\begin{array}{l}5.3 \\
0.71\end{array}$ & 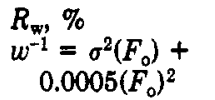 & 6.54 \\
\hline
\end{tabular}

the benzene derivatives $\mathrm{C}_{6}\left(\mathrm{CHR}_{2}\right)_{6}(\mathrm{R}=\mathrm{Cl}, \mathrm{Me})^{10}$ and very recently, for the $\left[\mathrm{C}_{5}{ }^{i} \mathrm{Pr}_{5}\right]$ radical. ${ }^{11}$ The $\mathrm{C}_{5}$ ring in 7 shows no deviation from planarity. Two silicon atoms in 1,3 position are shifted out of this plane by $30 \mathrm{pm}$ away from the manganese atom, while the silicon atom in between is situated $12 \mathrm{pm}$ on the proximal side of the ring. Thus the steric interaction between the silyl groups becomes minimized.

7 contains not only a 5-fold silylated cyclopentadienyl ring but also five $\mathrm{Si}-\mathrm{H}$ groups that might be used for further functionalization. Complexes with the $\left[\mathrm{C}_{5} \mathrm{H}_{4^{-}}\right.$ $\left.\left(\mathrm{SiMe}_{2} \mathrm{H}\right)\right]$ ligand have been used in the hydrosilylation of alkynes $^{12}$ as well as of metal carbonyls. ${ }^{13}$ Use of com-

(10) Siegel, J.; Gutierrez, A.; Schweizer, W. B.; Ermer, O.; Mislow, K. J. Am. Chem. Soc. 1986, 108, 1569. Kahr, B.; Biali, S. E.; Schaefer, W.; Buda, A. B.; Mislow, K. J. Org. Chem. 1987, 52, 3713.

(11) Sitzmann, H.; Boese, R. Angew. Chem., Int. Ed. Engl. 1991, 30, 971.

(12) Dröesmar-Wolf, M. Gmelin Handbook of Inorganic Chemistry, 8th ed.; Organoiron Compounds Part A, Ferrocene 9; Springer-Verlag: Berlin, 1989; p 305.
Table II. Positional $\left(\times 10^{4}\right)$ and Equivalent Isotropic Thermal Parameters $\left(\AA^{2} \times 10^{3}\right)$

\begin{tabular}{|c|c|c|c|c|}
\hline & $x$ & $y$ & $z$ & $U(e q)$ \\
\hline $\mathbf{M n}$ & $1432(1)$ & $928(1)$ & 0 & $43(1)$ \\
\hline$C(1 A)$ & $1625(5)$ & $509(8)$ & $1581(8)$ & $37(2)$ \\
\hline$C(2 A)$ & $1279(5)$ & $1707(8)$ & $1498(8)$ & $39(2)$ \\
\hline$C(3 A)$ & $602(5)$ & 1478 (11) & $1023(10)$ & $40(2)$ \\
\hline$C(4 A)$ & $541(5)$ & $181(7)$ & $785(8)$ & $36(2)$ \\
\hline$C(5 A)$ & $1164(5)$ & $-454(10)$ & 1157 (9) & $42(2)$ \\
\hline $\mathrm{Si}(1 \mathrm{~A})$ & $2486(1)$ & $255(3)$ & $2182(3)$ & $53(1)$ \\
\hline $\operatorname{Si}(2 A)$ & $1570(2)$ & 3265 (3) & 1966 (3) & 53 (1) \\
\hline $\mathbf{S i}(3 \mathrm{~A})$ & $-149(2)$ & $2613(3)$ & $962(3)$ & $64(1)$ \\
\hline $\operatorname{Si}(4 A)$ & $-173(1)$ & $-648(3)$ & 101 (3) & 53 (1) \\
\hline Si(5A) & $1314(2)$ & $-2178(2)$ & $1280(3)$ & 57 (1) \\
\hline $\mathrm{C}(\mathrm{1B})$ & 1564 (15) & $57(26)$ & $1512(22)$ & $37(2)$ \\
\hline $\mathrm{C}(2 \mathrm{~B})$ & 1453 (15) & $1415(27)$ & $1602(22)$ & $39(2)$ \\
\hline$C(3 B)$ & 740 (18) & 1661 (32) & $1094(29)$ & $40(2)$ \\
\hline$C(4 B)$ & 475 (14) & $601(26)$ & $807(23)$ & $36(2)$ \\
\hline$C(5 B)$ & $958(16)$ & $-454(28)$ & $980(24)$ & $42(2)$ \\
\hline $\mathrm{Si}(1 \mathrm{~B})$ & 2308 (4) & $-780(8)$ & 2077 (7) & $56(3)$ \\
\hline $\mathrm{Si}(2 \mathrm{~B})$ & 2033 (4) & $2604(7)$ & $2176(7)$ & $53(3)$ \\
\hline $\mathbf{S i}(3 \mathrm{~B})$ & $294(5)$ & $3240(7)$ & $1284(7)$ & $59(3)$ \\
\hline $\mathrm{Si}(4 \mathrm{~B})$ & $-387(4)$ & $293(9)$ & $154(9)$ & $64(3)$ \\
\hline $\mathrm{Si}(5 \mathrm{~B})$ & $819(5)$ & $-2165(7)$ & $766(7)$ & $55(3)$ \\
\hline$C(11)$ & $3123(4)$ & $-725(9)$ & $1423(8)$ & $84(4)$ \\
\hline$C(12)$ & $2382(5)$ & $-356(9)$ & $3495(7)$ & 77 (3) \\
\hline$C(21)$ & $2349(5)$ & $3926(7)$ & $1375(8)$ & $74(3)$ \\
\hline$C(22)$ & $1631(6)$ & $3266(9)$ & $3391(7)$ & $87(4)$ \\
\hline$C(31)$ & $-74(5)$ & $4006(8)$ & $67(10)$ & $95(4)$ \\
\hline C(32) & $-366(5)$ & 3126 (9) & $2287(8)$ & $104(4)$ \\
\hline C(41) & $-938(4)$ & $-882(8)$ & $944(9)$ & $87(4)$ \\
\hline $\mathrm{C}(42)$ & $-395(5)$ & $42(9)$ & $-1159(7)$ & $85(4)$ \\
\hline$C(51)$ & $563(6)$ & $-2903(7)$ & $1962(9)$ & $95(4)$ \\
\hline$C(52)$ & $1523(5)$ & $-3035(7)$ & $71(9)$ & $89(4)$ \\
\hline$C(6)$ & $2313(4)$ & $1287(7)$ & $-234(6)$ & $59(3)$ \\
\hline$O(1)$ & $2886(3)$ & $1528(6)$ & $-409(6)$ & $96(3)$ \\
\hline$C(7)$ & $1438(5)$ & $-164(9)$ & $-998(6)$ & $67(3)$ \\
\hline$O(2)$ & $1456(4)$ & $-852(7)$ & $-1667(6)$ & $110(3)$ \\
\hline$C(8)$ & $1191(4)$ & $2094(8)$ & $-900(7)$ & 66 (3) \\
\hline$O(3)$ & $1040(3)$ & $2822(7)$ & $-1489(6)$ & $108(3)$ \\
\hline
\end{tabular}

pounds 3-7 in such reactions should result in a variety of interesting, novel polyfunctionalized cyclopentadienyl complexes. Compound 7 can be expected to be a key intermediate in the synthesis of persilylated cyclopentadienyl ligands.

\section{Experimental Section}

All reactions were carried out under nitrogen with standard Schlenk tube techniques. Solvents were dried according to standard procedures and saturated with nitrogen. For the

(13) E.g.: Colomer, E.; Corriu, R. J. P.; Pleixats, R. J. Organomet. Chem. 1990, 381, C1. 
Table III. Important Distances $(\AA)$ and Angles (deg) in 7

$\begin{array}{ccc}\mathrm{C}(1 \mathrm{~A})-\mathrm{C}(5 \mathrm{~A}) & 1.472(14) & \mathrm{C}(1 \mathrm{~A})-\mathrm{C} \\ \mathrm{C}(3 \mathrm{~A})-\mathrm{C}(4 \mathrm{~A}) & 1.426(14) & \mathrm{C}(4 \mathrm{~A})-\mathrm{C} \\ \mathrm{C}(2 \mathrm{~A})-\mathrm{Si}(2 \mathrm{~A}) & 1.864(9) & \mathrm{C}(3 \mathrm{~A})-\mathrm{S} \\ \mathrm{C}(5 \mathrm{~A})-\mathrm{Si}(\mathrm{5A}) & 1.873(11) & \\ \mathrm{C}(2 \mathrm{~A})-\mathrm{C}(1 \mathrm{~A})-\mathrm{C}(5 \mathrm{~A}) & \\ \mathrm{C}(2 \mathrm{~A})-\mathrm{C}(3 \mathrm{~A})-\mathrm{C}(4 \mathrm{~A}) & 108.0(8) \\ \mathrm{C}(4 \mathrm{~A})-\mathrm{C}(5 \mathrm{~A})-\mathrm{C}(1 \mathrm{~A}) & 109.2(9) \\ \end{array}$

chromatographic separations Kieselgel 60 from Merck was used. 2 was prepared from $\mathrm{Mn}(\mathrm{CO})_{5} \mathrm{Br}$ and $\mathrm{C}_{6} \mathrm{Br}_{4} \mathrm{~N}_{2}$ according to a literature procedure. ${ }^{5} n$-Butyllithium was purchased as a $1.6 \mathrm{M}$ solution in hexane from Aldrich. SiMes $\mathrm{HCl}$ (Fluka) was distilled from $\mathrm{CaH}_{2}$ under nitrogen. For details of the crystal structure determination, Table I. Positional and thermal parameters of all atoms are included in Table II; important distances and angles are in Table III.

Preparation of 3. With continuous stirring, $1.04 \mathrm{~mL}$ of $\mathrm{BuLi}$ solution (1.66 mmol) was added to a solution of $1.0 \mathrm{~g}$ of 2 (1.67 $\mathrm{mmol}$ ) in $20 \mathrm{~mL}$ of $\mathrm{Et}_{2} \mathrm{O}$ at $-76^{\circ} \mathrm{C}$. After $30 \mathrm{~min}, 0.18 \mathrm{~mL}$ of $\mathrm{SiMe}_{2} \mathrm{HCl}(1.67 \mathrm{mmol}$ ) was added, and then the temperature was gradually raised to ambient temperature during $14 \mathrm{~h}$. After evaporation of the solvent in vacuo, the residue was ertracted with herane, the extracts were evaporated, and the residue was chromatographed on silica gel $(2.5 \mathrm{~cm} \times 10 \mathrm{~cm}$, eluent hexane). Recrystallization of the eluate yielded $0.87 \mathrm{~g}$ of analytically pure product $(91 \%), \mathrm{mp} 100-102{ }^{\circ} \mathrm{C}$. ${ }^{1} \mathrm{H}$ NMR $\left(270 \mathrm{MHz}, \mathrm{C}_{6} \mathrm{D}_{6}\right) \delta=$ $0.215\left(\mathrm{~d},{ }^{3} J(\mathrm{H}, \mathrm{H})=3.9 \mathrm{~Hz}, 6 \mathrm{H}, \mathrm{SiCH}_{3}\right), 4.548$ (sept, ${ }^{3} J(\mathrm{H}, \mathrm{H})=$ $3.9 \mathrm{~Hz}, 1 \mathrm{H}, \mathrm{SiH}) .{ }^{13} \mathrm{C} \mathrm{NMR}\left(68 \mathrm{MHz}, \mathrm{C}_{6} \mathrm{D}_{6}\right) \delta=-2.7\left(\mathrm{SiCH}_{3}\right)$, 80.6, 90.2, 94.9 $\left(\pi-\mathrm{C}_{5} \mathrm{R}_{5}\right), 222.4(\mathrm{MnCO})$. IR (Nujol) $\nu\left[\mathrm{cm}^{-1}\right]=$ $2169 \mathrm{w}(\mathrm{Si}-\mathrm{H}), 2039 \mathrm{vs}, 1969$ vs $(\mathrm{MnC}-\mathrm{O}) . \mathrm{C}_{10} \mathrm{H}_{7} \mathrm{Br}_{4} \mathrm{MnO}_{3} \mathrm{Si}$ (577.80) (calcd/found) C 20.79/21.02, H 1.22/1.30.

Preparation of 4. A solution of $0.50 \mathrm{~g}$ of $2(0.84 \mathrm{mmol})$ in 10 $\mathrm{mL}$ of $\mathrm{Et}_{2} \mathrm{O}$ was treated with $1.05 \mathrm{~mL}$ of BuLi solution (1.68 mmol) at $-76^{\circ} \mathrm{C}$ with continuous stirring. After $35 \mathrm{~min}, 0.18 \mathrm{~mL}$ of $\mathrm{SiMe}_{2} \mathrm{HCl}(1.67 \mathrm{mmol})$ was added. The temperature was raised to $20^{\circ} \mathrm{C}$ during the course of $14 \mathrm{~h}$. Further workup was performed as described for 3 , yield $\left.0.45 \mathrm{~g}(92 \%), \mathrm{mp} 96-98^{\circ} \mathrm{C}\right) .{ }^{1} \mathrm{H}$ NMR $\left(270 \mathrm{MHz}, \mathrm{C}_{6} \mathrm{D}_{6}\right) \delta 0.279\left(\mathrm{~d},{ }^{3} \mathrm{~J}(\mathrm{H}, \mathrm{H}) 3.9 \mathrm{~Hz}, 6 \mathrm{H}, \mathrm{SiCH}_{3}\right), 0.312$ $\left(\mathrm{d}^{3} J(\mathrm{H}, \mathrm{H})=4.0 \mathrm{~Hz}, 6 \mathrm{H}, \mathrm{SiCH}_{3}\right), 4.697\left(\mathrm{sept},{ }^{3} J(\mathrm{H}, \mathrm{H})=3.9 \mathrm{~Hz}\right.$, $2 \mathrm{H}, \mathrm{SiH}) .{ }^{13} \mathrm{C} \mathrm{NMR}\left(100 \mathrm{MHz}, \mathrm{C}_{6} \mathrm{D}_{6}\right) \delta=-3.11,-2.43\left(\mathrm{SiCH}_{3}\right)$, 86.14, 97.15, $101.92\left(\pi-\mathrm{C}_{5} \mathrm{R}_{5}\right), 223.0(\mathrm{Mn}-\mathrm{CO}) . \mathrm{IR}$ (hexane) $\nu\left[\mathrm{cm}^{-1}\right]$ $=2169 \mathrm{w}(\mathrm{Si}-\mathrm{H}), 2035 \mathrm{vs}, 1966 \mathrm{vs}(\mathrm{MnC}-\mathrm{O}) . \mathrm{C}_{12} \mathrm{H}_{14} \mathrm{Br}_{3} \mathrm{MnO}_{3} \mathrm{Si}_{2}$ (557.05); C, 25.87/26.36; H, 2.53/2.61.

Preparation of 5. In a manner similar to the synthesis of 4, compound 5 is obtained from 3 by double lithiation: A solution of $0.58 \mathrm{~g}$ of $3(1.00 \mathrm{mmol})$ in $10 \mathrm{~mL}$ of $\mathrm{Et}_{2} \mathrm{O}$ was treated with 1.25 $\mathrm{mL}$ of BuLi solution $(2.00 \mathrm{mmol})$ at $-76^{\circ} \mathrm{C}$. After $30 \mathrm{~min}, 0.22$ $\mathrm{mL}$ of $\mathrm{SiMe}_{2} \mathrm{HCl}(2.01 \mathrm{mmol})$ was added. After workup, $0.42 \mathrm{~g}$ of 5 was obtained $(78 \%), \mathrm{mp} 62.5^{\circ} \mathrm{C},{ }^{1} \mathrm{H}$ NMR $\left(400 \mathrm{MHz}, \mathrm{C}_{6} \mathrm{D}_{8}\right)$ $\delta=0.250\left(\mathrm{~d},{ }^{3} J(\mathrm{H}, \mathrm{H})=3.7 \mathrm{~Hz}, 6 \mathrm{H}, \mathrm{SiCH}_{3}\right), 0.368\left(\mathrm{~d},{ }^{3} J(\mathrm{H}, \mathrm{H})\right.$ $\left.=3.9 \mathrm{~Hz}, 6 \mathrm{H}, \mathrm{SiCH}_{3}\right), 0.415\left(\mathrm{~d},{ }^{3} J(\mathrm{H}, \mathrm{H})=4.2 \mathrm{~Hz}, 6 \mathrm{H}, \mathrm{SiCH}_{3}\right)$ 4.723 (sept, $\left.{ }^{3} J(\mathrm{H}, \mathrm{H})=3.9 \mathrm{~Hz}, 2 \mathrm{H}, \mathrm{SiH}\right), 4.806$ (sept, ${ }^{3} J(\mathrm{H}, \mathrm{H})$ $=3.9 \mathrm{~Hz}, 1 \mathrm{H}, \mathrm{SiH}) .{ }^{13} \mathrm{C} \mathrm{NMR}\left(100 \mathrm{MHz}, \mathrm{C}_{6} \mathrm{D}_{6}\right) \delta=-1.74,-2.55$,

$-2.78\left(\mathrm{SiCH}_{3}\right), 89.57,94.20,105.62\left(\pi-\mathrm{C}_{5} \mathrm{R}_{5}\right), 223.6(\mathrm{Mn}-\mathrm{CO})$. IR (hexane) $\nu\left[\mathrm{cm}^{-1}\right]=2166 \mathrm{w}(\mathrm{Si}-\mathrm{H}), 2030 \mathrm{vs}, 1960 \mathrm{vs}, 1955 \mathrm{vs}$ (MnC-0). $\quad \mathrm{C}_{14} \mathrm{H}_{21} \mathrm{Br}_{2} \mathrm{MnO}_{3} \mathrm{Si}_{3}$ (536.30): C, 31.35/31.16; $\mathrm{H}$, 3.95/4.10.

Preparation of 6. Starting from 4,6 was prepared by double lithiation: $0.40 \mathrm{~g}$ of $4(0.72 \mathrm{mmol})$, dissolved in $10 \mathrm{~mL}$ of $\mathrm{Et}_{2} \mathrm{O}$, was treated with $0.90 \mathrm{~mL}$ of BuLi solution $(1.44 \mathrm{mmol})$ at -76 ${ }^{\circ} \mathrm{C}$. After addition of $0.16 \mathrm{~mL}$ of $\mathrm{SiMe}_{2} \mathrm{HCl}(1.44 \mathrm{mmol})$ and the usual workup procedure, $0.29 \mathrm{~g}$ of 6 was isolated $(78 \%)$, mp $101-103{ }^{\circ} \mathrm{C}$. ${ }^{1} \mathrm{H}$ NMR $\left(270 \mathrm{MHz}, \mathrm{C}_{6} \mathrm{D}_{6}\right) \delta=0.319\left(\mathrm{~d},{ }^{3} J(\mathrm{H}, \mathrm{H})\right.$ $\left.=3.9 \mathrm{~Hz}, 6 \mathrm{H}, \mathrm{SiCH}_{3}\right), 0.327\left(\mathrm{~d},{ }^{3} J(\mathrm{H}, \mathrm{H})=3.9 \mathrm{~Hz}, 6 \mathrm{H}, \mathrm{SiCH}_{3}\right)$, $0.402\left(\mathrm{~d},{ }^{3} J(\mathrm{H}, \mathrm{H})=3.9 \mathrm{~Hz}, 6 \mathrm{H}, \mathrm{SiCH}_{3}\right), 0.502\left(\mathrm{~d},{ }^{3} J(\mathrm{H}, \mathrm{H})=3.9\right.$ $\left.\mathrm{Hz} 6 \mathrm{H}, \mathrm{SiCH}_{3}\right), 4.562(\mathrm{~m}, 2 \mathrm{H}, \mathrm{SiH}), 4.676(\mathrm{~m}, 2 \mathrm{H}, \mathrm{SiH}) .{ }^{18} \mathrm{C}$ NMR (100 MHz, $\left.\mathrm{C}_{6} \mathrm{D}_{6}\right) \delta=-0.89,-1.15,-1.30,-2.37\left(\mathrm{SiCH}_{3}\right)$, $96.91,106.41,110.93\left(\pi-C_{5} R_{5}\right), 224.3(\mathrm{Mn}-\mathrm{CO})$. IR (hexane) $\nu$ $\left[\mathrm{cm}^{-1}\right]=2162 \mathrm{w}(\mathrm{Si}-\mathrm{H}), 2023 \mathrm{vs}, 1952 \mathrm{vs}, 1945 \mathrm{vs}(\mathrm{MnC}-\mathrm{O})$. $\mathrm{C}_{16} \mathrm{H}_{28} \mathrm{BrMnO}_{3} \mathrm{Si}_{4}$ (515.55): C, 37.27/36.98; $\mathrm{H}, 5.47 / 5.61$.

Preparation of 7. To a solution of $1.79 \mathrm{~g}$ of $5(3.33 \mathrm{mmol})$ in $20 \mathrm{~mL}$ of $\mathrm{Et}_{2} \mathrm{O}, 4.58 \mathrm{~mL}$ of BuLi solution ( $7.33 \mathrm{mmol}$ ) was added at $-76{ }^{\circ} \mathrm{C}$. After stirring for $45 \mathrm{~min}, 0.80 \mathrm{~mL}$ of $\mathrm{SiMe}_{2} \mathrm{HCl}(7.33$ mmol) was added and the temperature was raised to $20^{\circ} \mathrm{C}$ during the course of $14 \mathrm{~h}$. After the usual workup procedure, a yield of $1.40 \mathrm{~g}$ of light yellow crystals were obtained (85\%), mp 121-123 ${ }^{\circ} \mathrm{C}$. ${ }^{1} \mathrm{H}$ NMR $\left(400 \mathrm{MHz}, 25^{\circ} \mathrm{C}, \mathrm{C}_{6} \mathrm{D}_{6}\right) \delta=0.437\left(\mathrm{~d},{ }^{3} \mathrm{~J}(\mathrm{H}, \mathrm{H})=\right.$ $\left.3.9 \mathrm{~Hz}, 30 \mathrm{H}, \mathrm{SiCH}_{3}\right), 4.539\left(\mathrm{sept}^{3} \mathrm{~J}(\mathrm{H}, \mathrm{H})=3.9 \mathrm{~Hz}, 5 \mathrm{H}, \mathrm{SiH}\right)$. ${ }^{18} \mathrm{C} \mathrm{NMR}\left(68 \mathrm{MHz}, \mathrm{C}_{6} \mathrm{D}_{6}\right) \delta=-0.73\left(\mathrm{SiCH}_{3}\right), 109.32\left(\pi-\mathrm{C}_{5} \mathrm{R}_{6}\right), 225.0$ (Mn-CO). Low-temperature ${ }^{1} \mathrm{H}$ NMR (90 MHz, toluene- $d_{8}$ ): coalescence of the doublet at $0.45 \mathrm{ppm}$ is observed at $-80^{\circ} \mathrm{C}$, at $-90^{\circ} \mathrm{C}$ two broad signals with a distance of approximately $27 \mathrm{~Hz}$ can be seen. IR (hexane) $\nu\left[\mathrm{cm}^{-1}\right]=2157 \mathrm{w}(\mathrm{Si}-\mathrm{H}), 2017 \mathrm{vs}, 1940$ vs $(\mathrm{MnC}-\mathrm{O}) . \mathrm{C}_{18} \mathrm{H}_{35} \mathrm{MnO}_{3} \mathrm{Si}_{5}(494.80)$ : C, 43.69/43.33; $\mathrm{H}$, 7.13/6.98.

Acknowledgment. We are greatly indebted to Prof. W. Beck for providing us with laboratory space and financial support. This work also was supported by the Deutsche Forschungsgemeinschaft and the Fonds der Chemischen Industrie.

Supplementary Material Available: Tables of atomic coordinates, bond lengths, bond angles and anisotropic displacement coefficients (8 pages). Ordering information is given on any current masthead page.

OM920322E 